\title{
Size flips symmetry switch
}

\author{
Whether a cell divides symmetrically or asymmetrically during early development determines the fate of its \\ progeny. Now cell size has emerged as a key player in making this decision.
}

\section{Alexandra Jilkine}

A cell's polarity encodes the spatial differences in its shape and structure that allow it to develop into a multicellular animal. Specialized molecules that coordinate the establishment of this polarity are organized into distinct membrane domains, determining a geometric axis along which a cell undergoes division. When this division is asymmetric, it leads to different fates in the progeny of the cell. It would be natural to assume that cells use geometry to coordinate this process, but how this occurs remains a conceptual question of interest to both biologists and physicists. Now, reporting in Nature Physics, Lars Hubatsch and colleagues have shown ${ }^{1}$ that cell size affects the ability of the nematode worm Caenorhabditis elegans embryo to polarize - revealing a geometric basis for its early asymmetric divisions.

In its initial stages of development, the C. elegans embryo divides without cell growth, so each division results in a smaller cell. During this period, asymmetric divisions produce cells with different fates. Asymmetric divisions are thus essential for proper organism development. For example, the $\mathrm{P}$ cells, numbered P0 through P4, form the germline lineage comprising cells containing material that gets passed down to the organism's progeny (Fig. 1). The divisions of cells P0 through P3 are asymmetric, but once the $P$ cells reach a size threshold, symmetric cell divisions follow.

The future head and tail of a C. elegans worm is established when anterior-posterior polarity is set up in the embryo. This is achieved through the spatial segregation of the so-called PAR proteins, where PAR stands for partitioning defective. Previous experimental work has determined that the width of the PAR boundary interface separating the anterior and posterior domains in the cell is directly related to the diffusion and lifetime of PAR proteins on the membrane $e^{2}$ and is proportional to the square root of the diffusion coefficient. Hubatsch and colleagues showed that as the cells become smaller, this interface takes up an increasing fraction of the cell - eventually resulting in a loss of polarization.

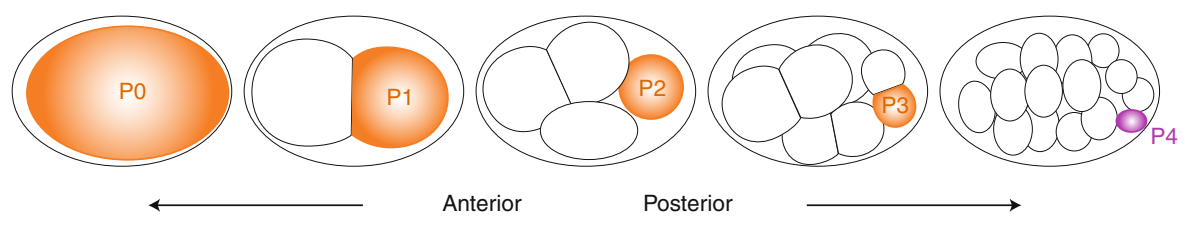

Fig. 1 | The P cells form the germline of the adult organism, and are created by asymmetric divisions during early development. The divisions of cells PO through P3 (orange) are asymmetric, but P4 (purple) divides symmetrically. Figure adapted from ref. ${ }^{8}$, under a Creative Commons licence (https://creativecommons.org/licenses/by/2.5/).

Mathematical models for interactions between diffusing polarity proteins have helped to elucidate the core design principles of cell polarity networks in different systems ${ }^{3}$. One key prediction from such models is that there are limits to the ability of pattern-forming systems to adapt to size changes ${ }^{4}$. Using numerical simulations of the PAR polarity network in C. elegans with experimentally measured parameters, Hubatsch and colleagues were able to predict a polarization size threshold that matches the size of the P3 cell, which is the smallest germline lineage cell to divide asymmetrically. They also applied physical and genetic perturbations known to affect embryo size, and showed that when these perturbations reduced the size of the $\mathrm{P} 3$ cell below the predicted threshold, the cell divided symmetrically.

Hubatsch and colleagues speculate that similar size-sensing mechanisms may be responsible for fate switches in other asymmetrically dividing lineages, such as embryonic neuroblasts in the fruit fly Drosophila. In other model systems for embryonic development, robust scaling of pattern with tissue size can be achieved through scaling the reaction rates ${ }^{5}$ of diffusing proteins. This effectively allows the organism to compensate for changes in its size, maintaining the correct proportions of its developing body plan.

Interestingly, there is some correlation between embryo size and the width of the interface set up by some - but not all PAR proteins. Specifically, there is a modest correlation between cell size and interface width for the PAR-2 proteins, which diffuse through the cell's posterior in the C. elegans embryo, but not for the anterior PAR-6 proteins. Why some biological systems adapt pattern to size, and some do not, remains unclear. The role that cortical and cytosolic flows ${ }^{6}$ and the clustering of PAR proteins both play in establishing and maintaining a robust anterior-posterior boundary also continues to be elucidated.

The importance of diffusion in establishing tissue-scale gradients in morphogenesis has been recognized for a long time. Through a combination of elegant experiments and simple theoretical analysis, Hubatsch and colleagues have now provided crucial support for the idea that cells can use diffusion on a cellular scale to inform decisions about cell fate.

\section{Alexandra Jilkine (D)}

Department of Applied and Computational Mathematics and Statistics, University of Notre Dame, Notre Dame, IN, USA.

e-mail: ajilkine@nd.edu

Published online: 12 August 2019 https://doi.org/10.1038/s41567-019-0644-z

\section{References}

1. Hubatsch, L. et al. Nat. Phys. https://doi.org/10.1038/s41567-0190601-x (2019).

2. Goehring, N. W., Hoege, C., Grill, S. W. \& Hyman, A. A. J. Cell Biol. 193, 583-594 (2011).

3. Halatek, J., Brauns, F. \& Frey, E. Phil. Trans. R. Soc. B 373 20170107 (2018).

4. Jilkine, A. \& Edelstein-Keshet, L. PLoS Comput. Biol. 7 , e1001121 (2011).

5. Ben-Zvi, D., Shilo, B. Z., Fainsod, A. \& Barkai, N. Nature 453, 1205-1211 (2008).

6. Wu, Y. et al. Proc. Natl Acad. Sci. USA 115, E8440-E8449 (2018).

7. Sailer, A., Anneken, A., Li, Y., Lee, S. \& Munro, E. Dev. Cell 35, 131-142 (2015)

8. Gönczy, P. \& Rose, L. S. in WormBook (ed. The C. elegans Research Community) https://doi.org/10.1895/wormbook.1.30.1 (2005). 